\title{
Avaliação da ingestão de minerais antioxidantes em idosos
}

Evaluation of antioxidant minerals intake in elderly

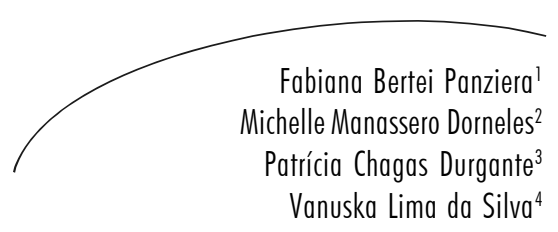

Resumo

Objetivo: Avaliar a ingestão de minerais antioxidantes em idosos e verificar níveis de adequação, bem como a correlação entre a ingestão desses minerais com o índice de massa corporal (IMC), sexo e idade. Metodologia: Realizou-se estudo com 51 idosos ( $\geq 60$ anos), atendidos no ambulatório de Geriatria e Gerontologia do Hospital São Lucas da PUC-RS. Aplicaram-se um questionário de frequência alimentar e um recordatório de 24 horas para avaliar os minerais ingeridos de acordo com a Ingestão Dietética de Referência (DRI), considerando os valores da ingestão adequada (AI), do requerimento médio estimado (EAR) e da ingestão dietética recomendada (RDA). Para avaliação antropométrica, utilizou-se o IMC. O recordatório de 24 horas foi analisado através do Programa de Apoio à Nutrição (Nutwin). Resultados: Neste estudo houve prevalência de mulheres $(78,4 \%)$. O IMC médio encontrado foi de $27,7 \mathrm{~kg} / \mathrm{m}^{2}$. O mineral que apresentou maior percentual de adequação foi o cobre $(54,9 \%)$ em relação às recomendações da RDA. Encontraram-se valores de ingestão de zinco e manganês inferiores às recomendações da EAR para a população feminina, respectivamente, $57,5 \%$ e $55 \%$; entretanto, valores de ingestão superiores às recomendações da RDA para estes mesmos minerais foram detectados na população masculina (45,5\% e 63,3\%). O selênio apresentou maior percentual de inadequação (98\%) e somente o manganês apresentou uma correlação inversa entre as variáveis, consumo e idade. Conclusão: $\mathrm{O}$ estudo identificou ingestão insuficiente dos minerais avaliados, sugerindo necessidade de maior atenção na prescrição dietética da população idosa, visto sua importância na prevenção de doenças, especialmente as crônicas não-transmissíveis.

\footnotetext{
Pós-Graduação em Nutrição Clínica e Estética. Instituto de Pesquisas, Ensino e Gestão em Saúde (IPGS). Porto Alegre, RS, Brasil.

2 Pós-Graduação em Nutrição Clínica. Universidade do Vale do Rio dos Sinos-(Unisinos). Porto Alegre, RS, Brasil.

3 Instituto de Geriatria e Gerontologia. Pontifícia Universidade Católica do Rio Grande do Sul (PUCRS). Porto Alegre, RS, Brasil.

4 Faculdade de Medicina, Curso de Nutrição. Universidade Federal do Rio Grande do Sul. Porto Alegre, RS, Brasil.
}

Palavras-chave: Estresse Oxidativo. Idosos.

Minerais na dieta.

Prevenção de doenças. 


\section{Abstract}

Objective: Assessing the intake of antioxidant minerals in elderly and checking the levels of adequacy, as well as the correlation between the ingestion of these minerals with the body mass index (BMI), sex and age. Methodology: A study was carried out with 51 elders, above 60 years old, at the Institute of Geriatrics and Gerontology from São Lucas's Hospital (PUC-RS). A food frequency questionnaire and a 24hour-recall were applied to evaluate the minerals intake in accordance with the Dietary Reference Intake (DRI), considering the adequate intake (AI), the estimated average requirement (EAR) and recommended dietary intake (RDA). To evaluate the anthropometric measures, the BMI were used. The 24 hourrecall was analyzed through the Nutrition Support Software (Nutwin). Results: In the studied group women predominated $(78,4 \%)$. The middle BMI was $27.7 \mathrm{~kg} / \mathrm{m}^{2}$. The copper was the mineral that presented higher percentage of adequacy $(54.9 \%)$, regarding the recommendations of the RDA. Inferior values of ingestion from zinc and manganese to the recommendations of the EAR were found in the feminine population, respectively, $57.5 \%$ and $55 \%$; however, values of ingestion higher than RDA recommendations for the same minerals were detected in the male population $(45.5 \%$ and $63.3 \%)$. Selenium was the mineral with higher percentage of inadequacy (98\%) and manganese was the only one that presented a reverse correlation between the variables, consumption and age. Conclusion: The study identified an insufficient ingestion of the evaluated minerals, showing the need for more attention in the dietetic prescription of the elderly population, knowing that they are very important in the prevention of some diseases, especially noncommunicable chronic diseases.
Key words: Minerals, Antioxidants, Oxidative Stress, Elderly.

\section{INTRODUÇÃO}

$\mathrm{Na}$ última década, o envelhecimento populacional tornou-se objeto de discussões e estudos, devido ao aumento progressivo de indivíduos nessa faixa etária. O envelhecimento humano é um fenômeno complexo, com o acúmulo de mudanças fisiológicas ocasionadas pelo tempo que conduzem o indivíduo a uma maior vulnerabilidade a doenças, principalmente às doenças crônicas não-transmissíveis e, com isso, ao desenvolvimento de incapacidades associadas a esse processo. ${ }^{1}$

Muitas teorias foram sugeridas para explicar esse processo. Dentre as mais aceitas, está a teoria baseada no estresse oxidativo, um desequilíbrio causado quando as defesas antioxidantes estão quantitativa ou qualitativamente impossibilitadas de neutralizar a produção e os efeitos de moléculas oxidantes. O estresse oxidativo teria influência decisiva sobre o envelhecimento humano, ocasionando danos em biomoléculas como lipídios, proteínas e DNA, que vão se acumulando ao longo dos anos, produzindo injúrias celulares e teciduais e, assim, levando ao envelhecimento do organismo. ${ }^{2}$

Estudos recentes têm chamado a atenção para certos nutrientes e componentes alimentares com atividade antioxidante, ou seja, com capacidade de transformar e/ou diminuir a ação de oxidação dos radicais livres, impedindo seus efeitos danosos ao organismo. A saber, o beta-caroteno, que no organismo se converte em vitamina $A$, as vitaminas $\mathrm{C}, \mathrm{E}$ e do complexo $\mathrm{B}$, como o ácido fólico e minerais como selênio, zinco, manganês e cobre. ${ }^{3}$

O cobre tem funções orgânicas específicas por ser constituinte de enzimas com atividade de oxiredução, como a cobre-zinco superóxido dismutase, que protege as células dos efeitos tóxicos no metabolismo do oxigênio; lisil oxidase, que catalisa a formação do colágeno e elastina; citocromo-c oxidase, necessária para o transporte 
de elétrons durante a respiração aeróbica; ceruplasmina, que é essencial para absorção e transporte de ferro necessário para a síntese de hemoglobina, entre outras. Também está envolvido no metabolismo do esqueleto, no sistema imunológico e na prevenção de doenças cardiovasculares. ${ }^{4}$

O selênio faz parte da enzima antioxidante, glutationa peroxidase, que atua no interior $\mathrm{da}$ célula convertendo compostos tóxicos, peróxido de hidrogênio, em atóxicos, água $\mathrm{e} \mathrm{O}_{2}$, resultando na redução de radicais livres. Também pode detoxificar metais pesados e substâncias carcinogênicas, além de estar envolvido no sistema de defesa do organismo, produzindo efeito protetor nos idosos pela modulação do sistema imunológico ${ }^{4}$. Sua ingestão tanto por indivíduos idosos quanto por adultos jovens, geralmente está inadequada, dando base para o uso de suplementos. Todavia, é incerto se a ingestão de selênio em doses maiores que os indicados pelas DRIs poderia produzir qualquer efeito benéfico na redução de risco de doenças não-transmissíveis. ${ }^{4}$

A atividade de diversas enzimas depende do metal-traço zinco. Entre elas está a superóxido dismutase. Acredita-se, assim, que esse micronutriente bloqueie a formação desses radicais livres, mesmo após a peroxidação celular já ter sido iniciada, através de sua presença do mecanismo de ativação de metaloproteínas celulares, protetoras de ações oxidativas. ${ }^{5}$

O manganês tem uma concentração mais significativa na mitocôndria do que em outras organelas celulares. Por fazer parte de duas metaloenzimas, a carboxilase piruvato e a Mnsuperóxido dismutase, sua presença na mitocôndria é de suma importância. Tem sido sugerido que a deficiência em Mn pode ter importante papel na peroxidação lipídica hepática. Alguns autores sugerem que a deficiência em Mn afeta o transporte da glicose e o metabolismo das células do adipócito. ${ }^{6}$

De um modo geral, os idosos são considerados o grupo mais mal nutrido de todos e há muitas razões para isso: o apetite, o paladar e o olfato diminuem com a idade, tornando a comida menos atraente. Muitas pessoas têm dificuldade de mastigar, além disso, azia, prisão de ventre, intolerância à lactose e outros problemas digestivos que aumentam com a idade. ${ }^{7}$

As mudanças fisiológicas decorrentes do envelhecimento interferem no estado nutricional, sendo elas: diminuição do metabolismo basal, redistribuição da massa corporal, alterações no funcionamento digestivo, alterações na percepção sensorial e diminuição da sensibilidade à sede. Com exceção das duas primeiras, todas as outras podem interferir diretamente no consumo alimentar, acarretando maior suscetibilidade desse grupo a estados de desnutrição e deficiências específicas de nutrientes. ${ }^{8}$

O estresse oxidativo e as deficiências nutricionais podem promover o aparecimento de diversas doenças crônicas associadas com o avanço da idade, como doença de Alzheimer, doença de Parkinson, esclerose, perdas cognitivas, além de doenças cardiovasculares. ${ }^{9}$

Considerando o importante efeito dos minerais antioxidantes na neutralização do estresse oxidativo e consequente prevenção de doenças, este estudo tem por objetivo avaliar o consumo alimentar desses minerais - a saber, cobre, zinco, selênio e manganês - em idosos atendidos em um ambulatório geriátrico, bem como analisar a correlação existente entre o IMC, sexo e faixa etária com os minerais acima citados.

\section{METODOLOGIA}

Foi realizado estudo observacional, descritivo e transversal, ${ }^{10}$ com 51 pacientes idosos, a partir de 60 anos, atendidos no ambulatório de geriatria do Instituto de Geriatria e Gerontologia do Hospital São Lucas da PUC-RS, os quais aceitaram participar voluntariamente da pesquisa, assinando o termo de consentimento livre e esclarecido previamente aprovado pelo Comitê de Ética em Pesquisa da PUC-RS. Parecer do Comitê de Ética n ${ }^{\circ}$ : CEP 08/04273 
Foram excluídos aqueles que não tiveram condições de responder ao questionário de frequência alimentar ou o inquérito recordatório alimentar de 24 horas.

O cálculo do tamanho da amostra foi realizado no programa PEPI (Programs for Epidemiologists) versão 4.0 e baseado em um estudo piloto com 15 idosos. Para um nível de confiança de $95 \%$, uma proporção de consumo de minerais antioxidantes abaixo do recomendado em $85 \%$ e uma margem de erro de $10 \%$, obteve-se um total mínimo de 49 indivíduos.

Para analisar os níveis de minerais antioxidantes consumidos pelos idosos, foi aplicado um questionário de frequência alimentar, previamente validado, ${ }^{11}$ o qual permitiu avaliar qualitativamente o consumo alimentar e um inquérito recordatório alimentar de 24 horas para avaliar quantitativamente os minerais ingeridos pelos idosos.

Para avaliação antropométrica, utilizou-se o índice de massa corporal (IMC), definido como: $\mathrm{IMC}=$ peso $(\mathrm{kg}) /$ altura $^{2}(\mathrm{~m})$ e classificou-se de acordo com Lipschitz ${ }^{12}$ para idosos. O peso foi verificado por meio de uma balança eletrônica da marca Welmy, com capacidade máxima de 200 $\mathrm{kg}$, devidamente calibrada; com o paciente descalço e com o mínimo de roupa possível, totalmente imóvel posicionado no centro $\mathrm{da}$ balança. Para a aferição da altura, utilizou-se um estadiômetro e o paciente foi orientado a ficar descalço, com os calcanhares unidos, com os braços relaxados e o mais ereto possível.

Ao final da entrevista, os idosos receberam orientação nutricional geral sobre alimentação saudável, Os Dez Passospara Uma Alimentacão Saudável para Pessoas Idosas. ${ }^{13}$ Os dados foram utilizados apenas para fins de pesquisa, não apresentando nenhum risco aos participantes, sendo garantido o anonimato dos mesmos.

As medidas caseiras foram convertidas em gramas e mililitros, para a análise quantitativa do que foi ingerido e o inquérito recordatório alimentar de 24 horas foi analisado através do Programa de Apoio à Nutrição (Nutwin) do Departamento de Informática da Universidade Federal de São Paulo (UNIFESP).

Para avaliação da adequação dos micronutrientes antioxidantes, foram utilizadas as recomendações das DRIs, considerando os valores da ingestão adequada (AI), do requerimento médio estimado (EAR) e da ingestão dietética recomendada (RDA), sendo que o único mineral avaliado pela AI foi o manganês, pois os outros já tem valores determinados de EAR. ${ }^{14}$

As variáveis foram descritas através de frequências absolutas e relativas. Para avaliar a associação entre as variáveis, o teste qui-quadrado de Pearson ou Exato de Fisher foram aplicados.

O nível de significância adotado foi de 5\%, sendo considerados estatisticamente significativos valores de $\mathrm{p}<0,05$ e as análises foram realizadas no programa SPSS (Statistical Packeage for the Social Sciences) versão 13.0.

\section{RESULTADOS}

A amostra foi constituída por 51 idosos, sendo em sua maioria mulheres $(78,4 \%)$ e concentrada principalmente na faixa etária dos 70 - 79 anos (43,1\%). A média de idade da amostra era de 72,2 anos. O IMC médio foi de $27,7 \mathrm{Kg} / \mathrm{m}^{2} \pm 5,5 \mathrm{de}$ desvio padrão. A caracterização da população da amostra com relação ao sexo, faixa etária e IMC está representada na tabela 1. 
Tabela 1 - Caracterização da população amostral, segundo sexo, faixa etária e IMC. Porto Alegre, RS, 2008.

\begin{tabular}{lcc}
\hline & $\mathrm{N}=51$ & $\%$ \\
\hline SEXO & 11 & 21,6 \\
Masculino & 40 & 78,4 \\
Feminino & & 37,3 \\
FAIXA ETÁRIA & 19 & 43,1 \\
60-69 anos & 22 & 19,6 \\
$70-79$ anos & 10 & 13,7 \\
$\geq 80$ anos & 7 & 33,3 \\
IMC (Kg/m $\left.{ }^{2}\right)$ & 17 & 52,9 \\
Magreza & 27 & \\
Eutrofia & & \\
Excesso de peso & & \\
\hline
\end{tabular}

O consumo alimentar dos minerais antioxidantes está descrito na tabela 2.

Tabela 2 - Avaliação da ingestão dos minerais. Porto Alegre, RS, 2008.

\begin{tabular}{|c|c|c|c|c|}
\hline \multicolumn{2}{|c|}{ CONSUMO MÉDIO } & HOMENS & MULHERES & \\
\hline \multicolumn{2}{|c|}{ Cobre (mg/dia) } & 1,31 & 0,95 & \\
\hline \multicolumn{2}{|c|}{ Zinco (mg/dia) } & 11,04 & 7,10 & \\
\hline \multicolumn{2}{|c|}{ Selênio ( $\mu \mathrm{g} / \mathrm{dia})$} & 20,14 & 0,31 & \\
\hline \multicolumn{2}{|c|}{ Manganês (mg/dia } & 3,36 & 1,65 & \\
\hline \multicolumn{2}{|c|}{ \% ADEQUAÇÃO } & $<$ EAR/AI $(\%)$ & EAR-RDA (\%) & > RDA/AI (\%) \\
\hline \multicolumn{2}{|l|}{ Cobre } & 31,3 & 13,7 & 54,9 \\
\hline \multirow[t]{2}{*}{ Zinco } & $\mathrm{M}$ & 55,0 & 7,5 & 37,5 \\
\hline & $\mathrm{H}$ & 36,3 & 18,1 & 45,4 \\
\hline \multicolumn{2}{|l|}{ Selênio } & 98,0 & 0,0 & 2,0 \\
\hline \multirow[t]{2}{*}{ Manganês } & $\mathrm{M}$ & 57,5 & 0,0 & 42,5 \\
\hline & $\mathrm{H}$ & 36,3 & 0,0 & 63,6 \\
\hline
\end{tabular}

Nota: $\mathrm{N}=51$ pacientes. Valores considerados de acordo com a EAR e AI.

O selênio obteve $98 \%$ de inadequação, apresentando valores abaixo do preconizado pela EAR. Na amostra, respectivamente, $55 \%$ e $57,5 \%$ das mulheres ingeriam quantidades inferiores de zinco e manganês, segundo a EAR/AI, enquanto que os homens em relação a esses mesmos minerais apresentaram um consumo acima da RDA, alcançando 45,4\% e 63,6\%. O cobre alcançou aproximadamente $55 \%$ de adequação, apresentando consumo superior ao recomendado pela RDA. Quando cruzado os dados, o único mineral que apresentou correlação significativa foi o manganês, o qual obteve uma correlação inversa importante entre o seu consumo e a faixa etária; $\mathrm{p}<0,05$ (figura 1). 


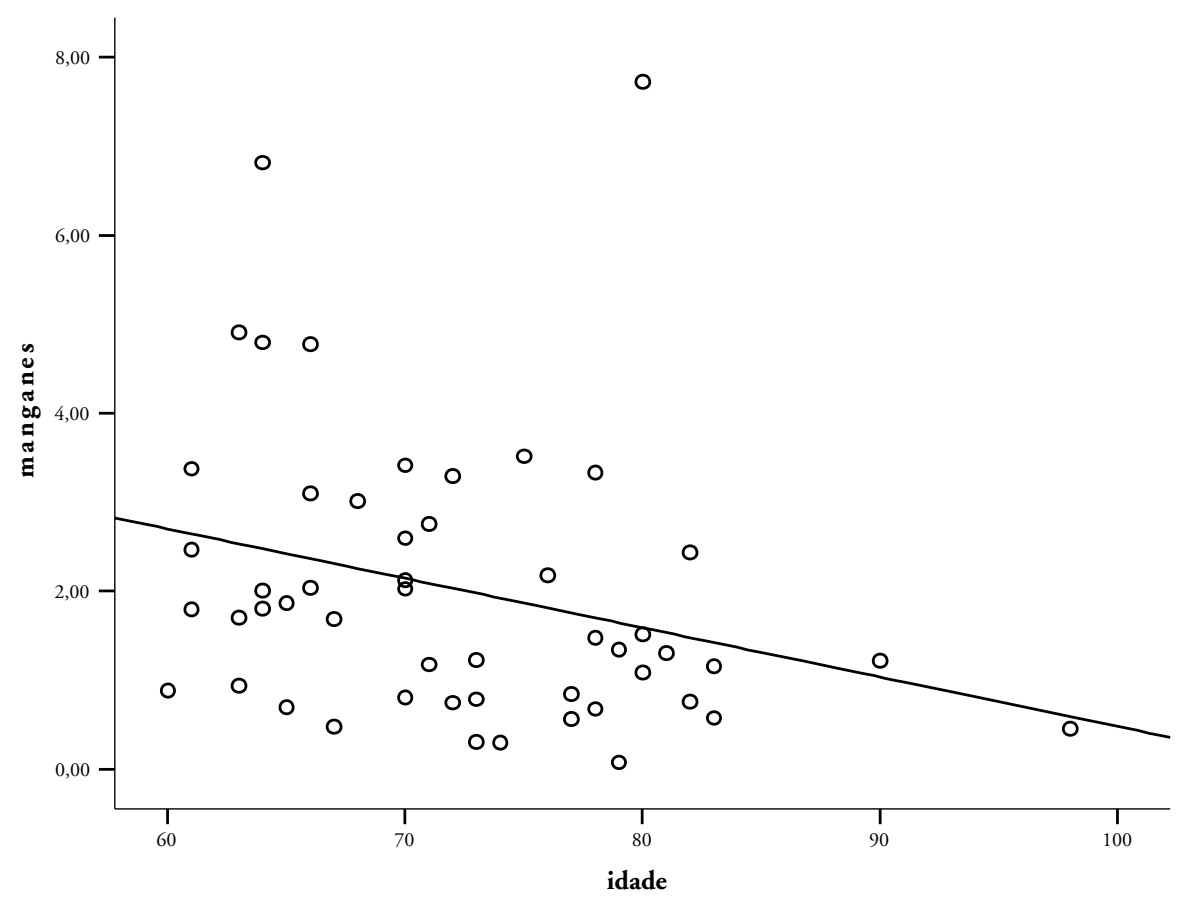

Figura 1 - Correlação entre a ingestão de manganês $(\mathrm{mg} / \mathrm{dia})$ e a idade (anos) $(\mathrm{p}<0,05)$.

Em relação ao grupo dos alimentos fonte de selênio e zinco, os alimentos protéicos como a carne, o frango, o peixe e o leite foram os que mais se destacaram. O peixe obteve seu maior consumo uma vez por semana $(25,5 \%)$, já as carnes $(45,1 \%)$ e o frango $(56,9 \%)$ foram consumidos em sua maioria de $2-4$ vezes por semana. O leite foi o que apresentou maior consumo (2-3 vezes no dia), atingindo um percentual de $74,5 \%$. (Figura 2)

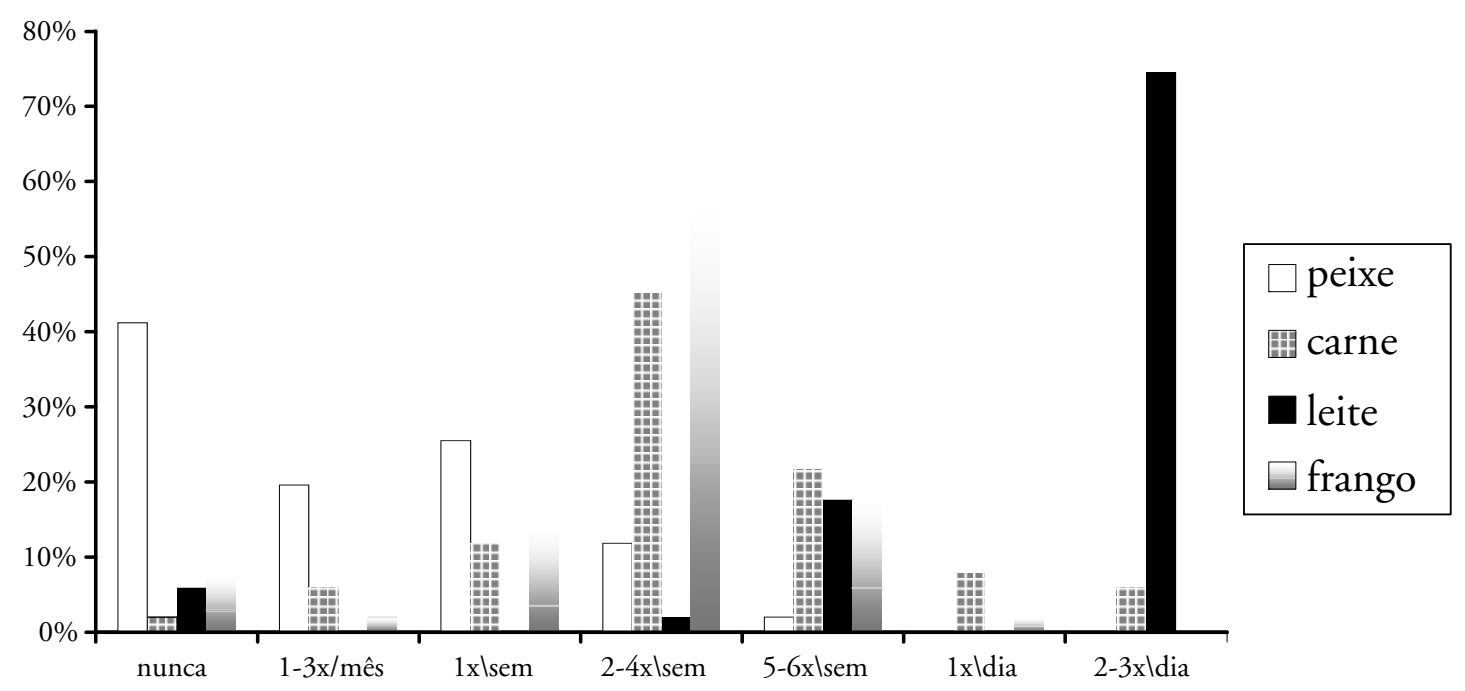

Figura 2 - Análise do questionário de frequência alimentar para fontes de selênio e zinco. 
Quanto aos alimentos fonte de cobre e manganês, o feijão foi o representante mais consumido do grupo das leguminosas, teve uma prevalência de consumo de $33,3 \%$ de $2-4$ vezes por semana.
Destaca-se o consumo de café pela população idosa (95\%), sendo ingerido principalmente de 2-3 vezes no dia. Vale ressaltar que mais de $50 \%$ relataram utilizar adoçante. De um total de 51 pacientes, 24 utilizavam açúcar; um açúcar mascavo, e 26, adoçante. (figura 3)

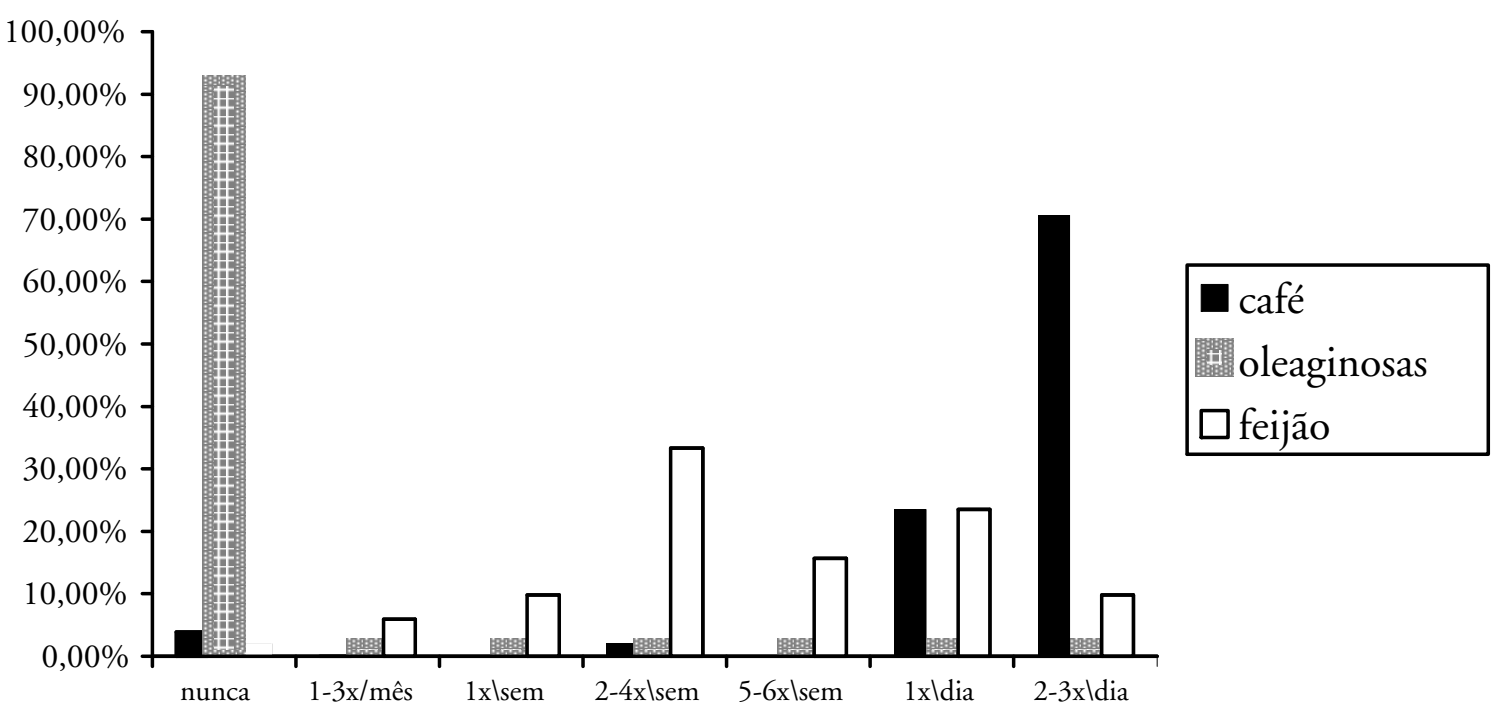

Figura 3 - Análise do questionário de frequência alimentar para fontes de cobre e manganês.

Também se verificou um elevado índice de participantes que relataram nunca consumirem outros alimentos fonte dos minerais estudados, como as oleaginosas $(92,2 \%)$, os frutos do mar $(100 \%)$ e o fígado $(80 \%)$.

Não houve correlação entre o consumo dos minerais estudados com o IMC, nem sexo; entretanto, quando associados com a faixa etária, o manganês foi o único mineral a apresentar correlação inversa significativa relevante, $\mathrm{p}<0,05$.

\section{DISCUSSÃO}

O percentual da população idosa que apresentou ingestão dos minerais antioxidantes estudados dentro dos padrões de adequação recomendados foi baixo, sendo esse desequilíbrio dietético caracterizado pela ingestão insuficiente desses. As inadequações apresentadas constituem um problema importante de saúde pública, pois além de os idosos serem considerados o grupo populacional mais suscetível a problemas nutricionais, ${ }^{15}$ existe uma associação entre o consumo desses minerais e o aparecimento de doenças crônicas relacionadas com o avanço da idade. ${ }^{9,3}$

A população do presente estudo foi caracterizada por um excesso de peso. No início da década de 90 , a frequência de baixo-peso atingia $20,75 \%$ nos homens e $17 \%$ nas mulheres. Em números absolutos seria um milhão e $300 \mathrm{mil}$ idosos com baixo-peso. Os idosos de baixa renda foram os mais atingidos, pois à medida que aumentava a renda per capita, reduzia-se o percentual de baixo-peso. O sobrepeso e sobretudo a obesidade parecem afetar, proporcionalmente mais as mulheres do que os homens. Praticamente metade da população idosa brasileira possuía excesso de peso, em todas as 
regiões do Brasil. ${ }^{16}$ Atualmente, estudos têm demonstrado um aumento no excesso de peso em ambos os sexos. ${ }^{17-19}$

Existem controvérsias quanto ao significado da obesidade entre os idosos e seu impacto, o qual parece ser menor do que o observado para adultos quanto à mortalidade. ${ }^{20}$ Por outro lado, o estudo realizado por Grabowski \& Ellis analisou a associação entre obesidade e mortalidade em idosos americanos, e verificou que essa condição, comparada à magreza e à manutenção do peso na faixa de normalidade, pode ser protetora para a ocorrência da mortalidade. ${ }^{21}$

O cobre é um mineral amplamente distribuído nos alimentos, o que torna mais rara sua deficiência. O presente estudo sugere que o cobre foi o mineral que apresentou maior percentual de adequação, pois praticamente $55 \%$ da população da amostra atingiram a recomendação segundo a RDA, configurando uma baixa probabilidade de inadequação.

A baixa ingestão de selênio constatada na população em estudo pode ser explicada pela enorme variabilidade desse mineral segundo a região. Neste estudo, apenas $2 \%$ estavam na faixa de adequação, reforçando a necessidade de intervenções, uma vez que a quantidade de selênio pode ser atingida apenas com o consumo de duas castanhas por dia, o qual foi referido apenas por um participante do estudo.

Os teores de selênio são mais elevados nos produtos de origem animal, sobretudo nos pescados, do que nos alimentos de origem vegetal consumidos no Brasil. Observa-se que, em razão dos baixos teores de selênio nos alimentos, para atingir a ingestão diária recomendada para um indivíduo adulto do sexo masculino, ${ }^{22}$ seria necessário ingerir alimentos em quantidades incompatíveis com a capacidade gástrica de um indivíduo adulto normal. Isso explica o fato da baixa adequação desse mineral, mesmo tendo se encontrado um alto consumo diário de leite, bem como semanal de carne, frango e peixe.

Em relação ao manganês, salienta-se que o percentual de adequação difere entre os sexos.
Destaca-se que a maioria das mulheres apresentou ingestão deste mineral abaixo da recomendação da $\mathrm{AI}$, enquanto que a maioria dos homens atingiu as recomendações da $\mathrm{AI}$ - portanto, não se pode determinar adequação no grupo das mulheres e se afirmar ingestão média adequada entre os homens.

O manganês foi o único mineral que, quando associado com a idade, apresentou significância relevante $(\mathrm{p}<0,05)$, uma correlação negativa leve, demonstrando que quanto mais jovem maior é o consumo do mineral. Sabendo-se que o manganês faz parte de uma metaloenzima, a Mn-superóxido dismutase e que sua ingestão é de suma importância, tem sido sugerido que a deficiência em manganês pode ter importante papel na peroxidação lipídica hepática. ${ }^{6}$

Considerando que o café é um alimento fonte de manganês e sabendo que ele se destacou entre os alimentos ingeridos pelos idosos, chegando a atingir $95 \%$ de consumo, pode-se dizer que ele foi crucial na oferta do mineral em questão, haja vista que o consumo de nozes foi praticamente nulo, e o das leguminosas, regular. Um percentual considerável de idosas referindo consumir café diariamente também foi observado por Marucci, ${ }^{23}$ sendo o item citado por todos os idosos.

Vale ressaltar que o zinco, da mesma forma que o manganês, tem recomendações diferentes entre os sexos e apresentou ingestão insuficiente de acordo com o preconizado pela EAR no grupo das mulheres, sugerindo implementação de intervenções para reverter este quadro. Já os homens, em sua maioria, atingiram a recomendação da RDA, demonstrando baixa probabilidade de inadequação.

A baixa ingestão de zinco tem sido associada à ingestão elevada de alimentos ricos em carboidratos, com pequena contribuição de proteína animal, um perfil comum entre idosos devido à menor renda e restrições para obter e preparar as refeições. ${ }^{24}$ Esses dados foram confirmados com os achados deste estudo, uma vez que se obteve um alto consumo de pão $(90 \%)$ diário, enquanto que os alimentos protéicos fonte dos minerais estudados, como a carne, o frango e os peixes, apresentaram um consumo semanal regular. 
O percentual de adequação do zinco encontrado neste estudo pode ser explicado pelo consumo diário de leite $(74,5 \%)$, sendo essa fonte alimentar o principal fator que contribuiu para elevar a ingestão diária desse mineral.

Finalmente, o perfil de consumo de nutrientes apresentado pela população se aproxima de outros estudos realizados em capitais e regiões metropolitanas do país. Um estudo que visava a analisar a ingestão dietética dos idosos verificou que $64,3 \%$ de idosos ( $\geq 60$ anos) relataram baixa ingestão protéica e $39,3 \%$ apresentavam inadequação das frações lipídicas, sendo que $35,7 \%$ informaram consumo excessivo de ácidos graxos saturados. Reduzidos níveis de cálcio, magnésio, zinco e cobre, como de vitamina D, C, E, B6, B1 e ácido fólico, foram identificados. ${ }^{25}$

Verifica-se, com frequência, elevado consumo de produtos industrializados, como doces e massas, ou de fácil preparo, como chás e torradas. Essa modificação no comportamento alimentar pode afetar a adequação de nutrientes no

\section{REFERÊNCIAS}

1. GIL L, et al. Age-associated analysis of oxidative stress parameters in human plasma and erythrocytes. Free Radical Research 2006;40(5):495-505.

2. Finkelt T, Holbrook NJ. Oxidants, oxidative stress and the biology of ageing. Nature 2000; 408: 239-247.

3. Meydani M. Nutrition interventions in aging and age-associated disease Ann. N Y Sci 2001;928 :226-235.

4. Cozzolino SMF. Biodisponibilidade de nutrients. 2 .ed .Barueri: Manole; 2007. 991 p.

5. Fortes C, et al. Zinc supplementation and plasma lipid peroxides in an elderly population. Eur J. Clin Nutr 1997 ;51(2):97-101.

6. Baly DL, Scheideman JS, Garcia-Welsh AL. Effect of manganese defiency on insulin binding, glucose transport and metabolism in rat adipocytes. J. Nutr 1990; 120(9):1075-9.

7. Saúde e Qualidade de Vida: Idoso. Rg Nutri ; São Paulo: [entre 2005-2008]. Disponível em: URL: http://www.rgnutri.com.br/sqv/idosos/ idoso.shtml. organismo dos idosos e os coloca em risco de má nutrição. ${ }^{26}$

\section{CONCLUSÃO}

Com base nos resultados obtidos, evidencia-se a baixa ingestão dos minerais antioxidantes estudados - a saber, cobre, zinco, selênio e manganês - nesse grupo. Isso pode estar relacionado tanto a fatores dietéticos, como a fatores fisiológicos do envelhecimento. Esses minerais são importantes antioxidantes, que auxiliam na prevenção de doenças crônicas - portanto, deve-se ter uma atenção especial na oferta destes quando feito o aconselhamento dietético aos idosos.

O número de idosos avaliados pode ser considerado uma limitação deste estudo, mas acreditamos que o resultado ainda fornece informações importantes. A realização de estudos específicos à população idosa pode direcionar atividades de promoção e recuperação da saúde por meio de adoção de hábitos alimentares saudáveis.
8. Clarke R, et al. Screening for vitamin B-12 and folate deficiency in older persons. Am. J. Clin. Nutr 2003; 77(5):1241-7.

9. Harman D. Free radical theory of aging: effect of free radical inhibitions on the mortality rate of male LFA mice. J. Gerontol 1968;23:476-482.

10. Goldim JR. Manual de Iniciação à Pesquisa em Saúde. 2 ed. Porto Alegre: Dacasa Editora; 2000.

11. Lopes C, et al. Consumo Alimentar no Porto. Faculdade de Medicina da Universidade do Porto 2006. Disponível em: URL: www.consumoalimentarporto.med.up.pt.

12. Lipshitz DA. Screening for nutritional status in the elderly. Prim Care 1994; 21(1):55-67.

13. Ministério da Saúde (Brasil). Cadernos de Atenção Básica: Envelhecimento e Saúde da Pessoa Idosa. Brasília 2006 ;19:180-184. Disponível em: URL: http:// dtr2004.saude.gov.br/dab/docs/publicacoes/ cadernos_ab/abcad19.pdf

14. Institute of Medicine. DRIs - Dietary Reference Intakes: Applications in Dietary Planning. National Academy Press. Washington, D.C 
2003. Disponível em: URL: http://

www.nap.edu.

15. Holleland G, et al. Cobalamin deficiency in general practice: assessment of the diagnostic utility and cost-benefit analysis of methylmalonic acid determination in relation to current diagnostic strategies. Clin Chem 1999; 45:189-198.

16. Coitinho DC, et al. Pesquisa nacional sobre saúde e nutrição: condições nutricionais da população brasileira - adulto e idoso. Brasília : INAN;1991; p.39.

17. Chaar CFS. Avaliação nutricional e alimentar de idosos matriculados do Centro de saúde. Rev. da Área de Ciências Biológicas e da Saúde 1996;1(1):12-15.

18. Frank AA. Estudo antropométrico e dietético de idosos. Rio de Janeiro [Dissertação]; Universidade Federal do Rio de Janeiro;1996.

19. Pereira RA. Avaliação antropométrica do estado nutricional. In: Sichieri R. Epidemiologia da obesidade. Rio de Janeiro : UERJ; 1998. (4): 43-63.

20. Visscher TLS, et al. A comparasion of body mass index, waist-hip ratio and waist circumference as predictors of all-cause mortality among the elderly: the Rotterdam study. Int J Relat Metab Disord 2001;25(11):1730-5.

21. Grabowski DC, Ellis JE. High body mass index does not predict mortality in older people: anaysis of the longitudinal study of aging. J Am Geriatr Soc 2001;49(7):968-79.

22. Ferreira $\mathrm{K}$, et al. Concentrações de selênio em alimentos consumidos no Brasil. Rev. Panam. Salud. Pública 2002;11(3).

23. Marucci MFN. Aspectos nutricionais e hábitos alimentares de idosos matriculados em ambulatório geriátrico. São Paulo [Tese ] Faculdade de Saúde Pública, Universidade de São Paulo;1992.

24. Sandstrôm B. Bioavailability of zinc. Eur J Clin Nut 1997; 51(Suppl 1):S17-9.

25. Lopes ACS, et al. Consumo de nutrientes em adultos e idosos em estudo de base populacional: Projeto Bambuí. Cad. Saúde Pública 2005 ;21(4).

26. Nogués R. Factores que afectan la ingesta de nutrientes en el anciano y que condicionan su correcta nutrición. Nutrición Clínica 1995; 15(2):39-44. 